\title{
Efecto de la adición de quinua (Chenopodium quinoa wild) malteada y sin maltear en la elaboración de cerveza tipo Ale a base de cebada (Hordeum vulgare) malteada
}

\author{
(Effect of the addition of malted and unmalted quinoa \\ (Chenopodium quinoa wild) in Ale type beer with barley \\ (Hordeum vulgare) malt)
}

\author{
Castañeda Ricardo ${ }^{1}$, Andrade-Cuvi María Josée ${ }^{2}$ Argüello Yolanda ${ }^{1}$, Vernaza María \\ Gabriela $^{3}$
}

\begin{abstract}
Resumen:
Se evaluó el efecto de la adición de quinua malteada (QM) y sin maltear (QSM) en la elaboración de cerveza tipo Ale a base de cebada malteada. Se elaboraron cervezas con $35 \%$ y $50 \%$ de QM y QSM. Previamente la quinua fue desaponificada y caracterizada. Antes y después de la fermentación se determinó la densidad, grado alcohólico, turbidez, acidez total y $\mathrm{pH}$. En el producto final se realizaron medidas de color $\left(\mathrm{L}, \mathrm{a}^{*}, \mathrm{~b}^{*}\right.$, Hue y $\left.\mathrm{Cr}^{*}\right)$ y se determinó la aceptabilidad sensorial. Respecto a la quinua cruda, el proceso de desaponificación aumentó la humedad, redujo la grasa y ceniza y ligeramente disminuyó los carbohidratos, el contenido de proteína y fibra no se vieron afectados. Luego de la fermentación la cerveza con QM presentó menor densidad, mayor grado alcohólico y turbidez. No se encontró diferencia en la acidez total y el $\mathrm{pH}$ mientras que el color de la cerveza con QM presentó menores valores de todos los parámetros de color analizados (excepto $\mathrm{a}^{*}$ ). La formulación con mayor aceptación global fue con 50\% QM. El uso de QM para la elaboración de cerveza constituye una alternativa para el procesamiento de este cereal y un producto novedoso para el consumidor.
\end{abstract}

Palabras clave: cerveza de alta fermentación; quinua malteada; análisis sensorial.

\begin{abstract}
:
The effect of the addition of malted quinoa (QM) and without malting (QSM) was evaluated in an Ale type beer with malted barley. Formulations containing 35 and $50 \%$ of QM and QSM were developed. Quinoa was previously unsapponificated and characterized. Density, alcoholic degree, turbidity, total acidity and $\mathrm{pH}$ were determined before and after the fermentation process. Analysis of color $\left(\mathrm{L}, \mathrm{a}{ }^{*}, \mathrm{~b}\right.$ *, Hue and $\left.\mathrm{Cr}{ }^{*}\right)$ and sensorial acceptability were performed. Unsapponification process increased moisture content, reduced fat and ash and slightly decreased carbohydrates, nevertheless, protein and fiber content were not affected. After the fermentation, the beer with QM presented lower density, higher alcohol content and turbidity. No difference was found in total acidity and $\mathrm{pH}$, while the color of beer with $\mathrm{QM}$ presented lower values in the analyzed color parameters (except a *). The formulation with the highest overall acceptance was the one with $50 \%$ QM. The use of QM for brewing is an alternative for the consuming of this cereal and a novel product for the consumer.
\end{abstract}

Keywords: high fermentation beer; malted quinoa; sensory analysis.

\footnotetext{
1 Universidad Tecnológica Equinoccial, Facultad de Ciencias de la Ingeniería e Industrias, Quito, Ecuador (ricardoct91@hotmail.com, asetecal@gmail.com)

2 Universidad Tecnológica Equinoccial, Centro de Investigación de Alimentos - CIAL, Facultad de Ciencias de la Ingeniería e Industrias, Quito, Ecuador (mjandradecuvi@ute.edu.ec)

${ }^{3}$ Universidad San Francisco de Quito, Colegio de Ciencias e Ingeniería - El Politécnico, Quito, Ecuador (mgvernaza@usfq.edu.ec)
} 


\section{Introducción}

En Ecuador en los últimos años el consumo de quinua ha sido considerado como secundario o poco aprovechable debido a su bajo consumo per cápita (1/2 libra por persona al año), escasa superficie de cultivo y bajo interés aparente de la población por incrementar su producción y consumo. La investigación botánica y agronómica en el país, ha prestado escasa atención a este grano, que tampoco forma parte de la alimentación humana fuera de las sociedades rurales andinas (Rodríguez, 2016).

La elaboración de una cerveza que contenga quinua (Chenopodium quinoa Willd) puede contribuir con el plan de recuperación de alimentos autóctonos de Ecuador, no solo por la capacidad agrícola del país para el cultivo de quinua, sino también por el aporte nutricional de este alimento que actualmente es desaprovechado (Padulosi, 2011). El año 2013 fue declarado como "Año Internacional de la Quinua" por la FAO (Food and Agriculture Organization) en reconocimiento a los pueblos andinos que han mantenido, controlado y protegido la quinua como alimento sagrado, con el fin de preservar la tradición e incentivar el reconocimiento de su aporte nutricional.

El comercio mundial de quinua ascendió a aproximadamente 135 millones de dólares en 2012. Actualmente, el intercambio comercial de este producto se encuentra muy concentrado, tanto por origen como por destino. El $82.4 \%$ de las exportaciones mundiales se originan en tres países andinos: Bolivia, Ecuador y Perú. Los otros exportadores importantes son Estados Unidos (9.8\%) y la Unión Europea (7.5\%), aunque en estos dos casos gran parte de las ventas corresponden a reexportaciones (FAO, 2014).

Al 2014, la producción de quinua se ubicó en 192,506 toneladas solamente en los 3 países que mayormente la producen, con una superficie cosechada de 243,227 hectáreas (FAO, 2014). Específicamente en Ecuador, durante el año 2015, la quinua ocupó el puesto 24 en producción entre los principales cultivos del país, con 12,707 toneladas producidas y una superficie cosechada de 7,148 hectáreas (Monteros, 2016).

Para la elaboración de cerveza se requiere de azúcares que puedan ser fermentables por levaduras. El contenido de almidón en la quinua está en un rango de $58.1 \%$ a $64.2 \%$, el cual puede ser degradado en azúcares fermentables mediante la ayuda de enzimas activadas por el proceso de malteado. Además, la quinua es un grano que contiene cantidades calóricas superiores a los cereales como arroz, trigo y maíz. Sus propiedades nutricionales son extraordinarias y los usos alimenticios son ilimitados por su sabor y aroma (De Bruin, 2006).

Actualmente el mercado de cerveza corresponde al $1.4 \%$ de la población ecuatoriana, aproximadamente 1'895820 personas consumen cerveza regularmente (INEC, 2011), con un crecimiento $11 \%$ anual del 2006 al 2009, sin embargo, ha enfrentado caídas desde entonces, manteniéndose un crecimiento promedio de $4 \%$ anual (INEC, 2011).

Se denominan Ale a las cervezas elaboradas con levaduras que fermentan favorablemente a temperaturas más altas $\left(16-29^{\circ} \mathrm{C}\right)$ que las levaduras "Lager" $\left(7-15^{\circ} \mathrm{C}\right)$. Las cervezas tipo Ale también se denominan de fermentación alta por la posición superficial de las levaduras en el fermentador. Estas levaduras suben a la superficie junto con el CO2 que producen, se alimentan y reproducen mientras ascienden y generan sabores especiales en el líquido que las rodea. Con este tipo de levadura se puede fermentar entre 16 a $29^{\circ} \mathrm{C}$, pues la levadura solo puede vivir y reproducirse en este rango de temperatura. La mayoría de cervezas tipo Ale tienen sabores y aromas complejos, pueden percibirse sabores y aromas a flores, frutas, especias de un sinnúmero de matices y generalmente tienen un gusto al paladar mucho más complejo. La fermentación con levaduras Ale produce una cerveza generalmente muy aromática y con sabores afrutados muy diferentes a las cervezas tipo Lager. Además, este tipo de cervezas se fermentan durante un período corto (aproximadamente 2 semanas), y pueden consumirse a los pocos días de finalizar la fermentación (Bamforth, 2003). 
El objetivo de este trabajo fue estudiar el efecto de la adición de quinua malteada (QD) y quinua sin maltear (QSM) en la elaboración de una cerveza tipo Ale a base de cebada malteada y determinar la capacidad fermentativa y cualidades organolépticas que la quinua puede aportar en la cerveza.

\section{Metodología}

Se utilizó quinua cruda proveniente de la zona del Lago San Pablo, provincia de Imbabura, sierra norte del Ecuador. Se utilizó cebada malteada Weyermann Pale Ale (Alemania). Para el amargor y aroma se empleó lúpulo East Kent Goldings (Reino Unido). Se utilizó levadura cervecera Fermentis - Safeale S-04 y carragenato como agente clarificante.

Eliminación y determinación de saponinas. - Se empleó el método de desaponificación tradicional, lavando los granos de quinua con abundante agua hasta observar el desprendimiento de las capas superficiales del grano, donde se encuentran las saponinas (Nieto et al., 1998).

Para la determinación del contenido de saponinas se siguió el método espumoso establecido en la Norma INEN 1672 (Ecuador, 2013), basada en las propiedades tensoactivas de las saponinas, que al disolverse en agua y agitarse generan una espuma estable, cuya altura puede correlacionarse con el contenido de saponinas en los granos. El porcentaje de saponinas se calculó con la Ecuación 1.

Donde:

$$
P S=\frac{(0.646 \times h)-0.104}{\mathrm{~m} \times 10}
$$

Ps = porcentaje de saponinas

$\mathrm{h}=$ altura de la espuma en tubo de ensayo

$\mathrm{m}=$ masa de la muestra

Caracterización fisicoquímica de la quinua. - La quinua fue caracterizada por su humedad, proteína, grasa, ceniza, fibra bruta siguiendo metodologías descritas por la AOAC (2010). Los carbohidratos fueron calculados por diferencia.

Malteado de quinua. - Para realizar el proceso de malteado de la quinua se siguió la metodología descrita por García et al. (2004), con algunas modificaciones. Para el proceso de germinación, los granos de quinua fueron inmersos en agua potable por un período de 24 horas a temperatura ambiente $\left(15^{\circ} \mathrm{C}\right)$. Se utilizó una proporción grano:agua de $1: 3$ y se realizaron cuatro cambios de agua a $15^{\circ} \mathrm{C}$ (cada 6 horas). Al final de la etapa de remojo, los granos alcanzaron $45 \%$ de humedad. Pasadas las 24 horas de remojo se identificó que los granos habían desarrollado una plúmula de tamaño igual o mayor a un tercio del grano, momento en el cual se suspendió la germinación. Para detener la germinación, los granos germinados se secaron durante 3 días y se tostaron en horno industrial por 2 horas a $80^{\circ} \mathrm{C}$. García et al. (2004) recomiendan temperaturas de secado de maltas para cervezas tipo Ale de $60-95^{\circ} \mathrm{C}$ donde ocurren reacciones de oscurecimiento no enzimático de Maillard que producen sabores y aromas de cualidades en las cervezas.

Diseño experimental. - Para estudiar el efecto de la adición de quinua malteada y sin maltear en la elaboración de cerveza se aplicó un diseño unifactorial siguiendo las 4 formulaciones descritas en la Tabla 1. En todas las formulaciones se utilizó quinua desaponificada.

Elaboración de cerveza. - Se elaboró una cerveza Ale siguiendo los parámetros tradicionales de una cerveza British Pale Ale. El proceso se calculó para producir 20 litros de cerveza, utilizando $10 \mathrm{~kg}$ de cereales. Los granos de cebada y quinua fueron molidos manualmente utilizando un molino que se preparó con una apertura suficiente para que el grano se parta, pero no se triture y que finalmente los granos se partan parcialmente sin 
llegar a obtener la harina de ninguno de los cereales. Se conservaron las cáscaras de los granos malteados para ser utilizados como lecho filtrante (Kunze, 2006).

Tabla 1. Formulaciones de cerveza

\begin{tabular}{cc}
\hline Código & Tratamiento \\
\hline QSM50 & $50 \%$ quinua sin maltear $-50 \%$ cebada malteada \\
QSM35 & $35 \%$ quinua sin maltear $-65 \%$ cebada malteada \\
QM50 & $50 \%$ quinua malteada $-50 \%$ cebada malteada \\
QM35 & $35 \%$ quinua malteada $-65 \%$ cebada malteada \\
\hline
\end{tabular}

Una vez partidos los granos se realizó el proceso de maceración, para el cual se utilizó un macerador de acero inoxidable con chaqueta de calefacción para mantener la temperatura. La mezcla inicial estuvo constituida por $25 \mathrm{~L}$ de agua y $10 \mathrm{~kg}$ de cereales. Se mezcló la cebada malteada, la quinua y se añadió agua caliente a $70^{\circ} \mathrm{C}$; se mantuvo la temperatura de la mezcla $\left(64^{\circ} \mathrm{C}\right)$ por una hora, transcurrido este tiempo se elevó la temperatura hasta $76{ }^{\circ} \mathrm{C}$ y se mantuvo 5 minutos para detener el proceso enzimático (Zainashef y Palmer, 2007).

Posteriormente se realizó la recirculación, este procedimiento consiste en recircular el mosto a través de un medio filtrante a la misma temperatura que al final de la maceración. Se utilizó una malla filtrante que se colocó sobre el macerador y se recirculó manualmente el mosto con recipientes de acero inoxidable por 30 minutos. Este proceso se realizó con el fin de extraer todos los azúcares fermentables y sabores de los cereales (Zainashef y Palmer, 2007).

El mosto obtenido se mantuvo en ebullición durante 90 minutos. Se añadió el lúpulo para generar un amargor y aroma deseados para una cerveza Brisith Pale Ale de la siguiente manera: a los 30 minutos se adicionó $15 \mathrm{~g}$ de lúpulo, a los 55 minutos $7.5 \mathrm{~g}$, a los 75 minutos $7.5 \mathrm{~g}$ y a los 85 minutos $7.5 \mathrm{~g}$ (Zainashef y Palmer, 2007). Faltando 15 minutos para completar ebullición se añadió el carragenato como agente clarificante. El carragenato clarificó la cerveza al generar un sedimento (trub) más homogéneo en el recipiente de cocción por su interacción con las proteínas desnaturalizadas durante el hervor. Una vez finalizada la cocción del mosto, se enfrió rápidamente para mejorar la clarificación y medir su densidad, denominada en adelante como "densidad inicial" (Kunze, 2006). Para lograr una mejor clarificación del producto el mosto se enfrió en un recipiente de acero inoxidable inmerso en hielo.

Asimismo, 11 gramos de levadura cervecera Safeale S-04 fueron activados en 250 $\mathrm{ml}$ de agua mezclada con mosto a $30^{\circ} \mathrm{C}$. Posteriormente se enfrió la totalidad del mosto hasta los $20^{\circ} \mathrm{C}$, con el fin de crear un ambiente adecuado para la reproducción de las levaduras $\left(18-22^{\circ} \mathrm{C}\right)$. Finalmente, el mosto fue colocado en el fermentador y se añadió la levadura activada. La fermentación se llevó a cabo durante 7 días a temperatura entre 18 y $22{ }^{\circ} \mathrm{C}$ dentro de un contenedor herméticamente sellado con válvula de fermentación.

Una vez finalizada la fermentación de la cerveza se trasvasó a un segundo recipiente, extrayendo la mayor cantidad de cerveza y dejando el sedimento del fondo en el primer fermentador. La cerveza se enfrió por 2 días a $4{ }^{\circ} \mathrm{C}$, temperatura en la cual la levadura restante se inhibe por las bajas temperaturas y la cerveza alcanza mayor clarificación. Para el envasado de la cerveza se utilizaron botellas ámbar de $296 \mathrm{ml}$. La carbonatación fue causada naturalmente al añadir azúcar a la cerveza $(6 \mathrm{~g} / \mathrm{L})$. Las levaduras inhibidas aún vivas en la cerveza, utilizan el azúcar añadido para generar CO2 adicional y necesario para el producto final. Finalmente se utilizó una tapadora manual para colocar las tapas corona y atrapar el gas producido naturalmente (Daniels, 2000). El producto se mantuvo a $4^{\circ} \mathrm{C}$ hasta su consumo y posterior análisis. 


\subsection{Caracterización de las cervezas obtenidas}

Determinación de la densidad. - Para la determinar la densidad del mosto y de los productos obtenidos se utilizó un densímetro calibrado a $20^{\circ} \mathrm{C}$. Una vez finalizada la fermentación y antes de colocar la cerveza en el cuarto frío, se utilizó el densímetro para medir la densidad final, la cual indicará el grado de atenuación y la cantidad del alcohol producido por la fermentación (Bamforth, 2003).

Volumen de alcohol. - Para determinar el volumen de alcohol ( $\left.{ }^{\circ} \mathrm{GL}\right)$ de los productos se utilizó un alcoholímetro calibrado a $20^{\circ} \mathrm{C}$. Se calentaron las muestras de cerveza hasta alcanzar los $20^{\circ} \mathrm{C}$ y luego se dejaron reposar por 30 minutos hasta alcanzar la eliminación de la mayor cantidad de gas. La separación del gas carbónico evita que alcoholímetro se eleve por acción del gas (Kunze, 2006).

Color. - Para la determinación del color de la cerveza se utilizó un colorímetro para líquidos Konica Minolta modelo CR-400. Se realizaron mediciones en el espacio de color CIE L*a*b*. El sensor del equipo se sumergió en la muestra de cerveza. Para determinar los valores de croma y hue se utilizaron las ecuaciones 2 y 3 (Retting y Ah- Hen, 2014). Las medidas se realizaron por triplicado.

$$
\begin{gathered}
\text { hue }=\operatorname{Arctg}\left(\frac{b}{a}\right)^{0.5} \\
\text { croma }=\left(a^{2}+b^{2}\right)^{0.5}
\end{gathered}
$$

Donde:

$\mathrm{a}=$ coordenada de cromaticidad en la gama de rojo - verde

$\mathrm{b}=$ coordenada de cromaticidad en la gama amarillo - azul

Turbidez. - Se determinó la turbidez de las cervezas en unidades NTU (Unidades Nefelométricas de turbidez) utilizando un turbidímetro con muestras precalentadas descarbonatadas. Se realizó este procedimiento para determinar si el proceso de clarificación con carragenato funcionó de igual forma en las cuatro formulaciones (Olsen, 2003).

Acidez. - Se determinó según el método descrito en la norma INEN 2323 (Ecuador, 2002). La cerveza fue previamente desgasificada calentando el líquido hasta $20^{\circ} \mathrm{C}$. Una vez comprobada la ausencia de gas, se neutralizó con solución de $\mathrm{NaOH} 0,1 \mathrm{~N}$ hasta alcanzar un $\mathrm{pH}$ de 8.2. Los resultados se expresaron como acidez total (como ácido láctico) en $\mathrm{cm} 3$ de álcali 1,0 $\mathrm{N}$ necesarios para neutralizar $100 \mathrm{~g}$ de cerveza utilizando las ecuaciones 4 y 5 .

Acidez total $=\left(\frac{\mathrm{cm}^{3} \text { de } \mathrm{NaOH} 0.1 \mathrm{~N} \times 10}{\mathrm{~cm}^{3} \text { de cerveza } \times \text { gravedad específica cerveza }}\right)$

Acidez total (como ácido láctico $)=\left(\frac{\mathrm{cm} 3 \mathrm{de} \mathrm{NaOH} 0.1 \mathrm{~N} \times 0.9}{\mathrm{~cm}^{3} \text { cerveza } \times \text { gravedad específica cerveza }}\right)$

Análisis sensorial. - Se realizó un análisis de aceptabilidad de los productos obtenidos mediante una escala hedónica de 9 puntos, con valores numéricos desde 1 que significó "me disgusta mucho" hasta 9 que significó "me gusta mucho". En este análisis participaron 100 jueces no entrenados, adultos consumidores de cerveza. Se evaluó la transparencia, color, sabor, aroma, cuerpo, sabor residual y aceptabilidad global (Mattos, 2004).

Análisis estadístico. - Todas las mediciones se realizaron por triplicado. Los resultados obtenidos fueron analizados mediante un análisis de varianza (ANOVA) unifactorial y las medias fueron comparadas por la prueba de Tukey (95\% de confianza). Para el análisis sensorial se realizó un diseño de bloques completamente aleatorizado (DBCA), con el 95\% de confianza. Se utilizó el programa Statgraphics Centurión XV. 


\section{Resultados}

Contenido de saponinas. - La Tabla 2 muestra los resultados de altura de espuma en los tubos de ensayo y el porcentaje de saponinas calculado para las muestras de quinua sin lavar y quinua cruda desaponificada.

Tabla 2. Contenido de saponinas en quinua sin lavar y cruda desaponificada

\begin{tabular}{ccc}
\hline Quinua & Altura $(\mathrm{cm})$ & Contenido de saponinas (\%) \\
\hline Sin lavar & $2.00 \pm 0,00^{\mathrm{a}}$ & $0.24^{\mathrm{a}}$ \\
Cruda desaponificada & $0.30 \pm 0,14^{\mathrm{b}}$ & $0.01^{\mathrm{b}}$ \\
\hline
\end{tabular}

Letras distintas indican diferencia significativa entre las muestras

El contenido de saponinas en la quinua sin lavar fue de $0.24 \%$, mientras que para la quinua desaponificada fue de $0.01 \%$. La quinua puede ser clasificada de acuerdo con la concentración de las saponinas en quinua dulce cuando contiene menos del $0.11 \%$ en quinua amarga cuando contiene más del $0.11 \%$ de saponinas (Koziol, 1992), es decir que la materia prima utilizada en este estudio corresponde a un tipo de quinua dulce.

Asimismo, la reducción del contenido de saponinas fue satisfactoria mediante el método tradicional de lavado de granos descrito por Nieto et al. (1998), pues la desaponificación se dio casi en su totalidad. Las saponinas en el grano de quinua se encuentran en el pericarpio y otorgan un sabor amargo y en grandes cantidades pueden ser tóxicas. Además, a pesar de su buen contenido de proteínas, los aminoácidos de la quinua cruda no lavada no están totalmente disponibles debido a las saponinas, que interfieren con la utilización biológica de los nutrientes (FAO, 2011, Repo-Carrasco, Espinoza y Jacobsen, 2003). Por esta razón es importante eliminar esos compuestos antes de consumir o procesar la quinua.

Caracterización fisicoquímica de quinua. - En la Tabla 3 se muestran los resultados de la caracterización fisicoquímica de granos de quinua cruda y desaponificada. El proceso de desaponificado influye directamente en los nutrientes del grano de quinua. En un artículo de revisión esta sección no es necesaria. En su lugar se deben incluir tantas secciones cuantos subtemas sea necesario revisar.

Tabla 3. Caracterización fisicoquímica de quinua cruda y desaponificada

\begin{tabular}{ccc} 
& \multicolumn{2}{c}{ Quinua } \\
\cline { 2 - 3 } Parámetro (\%) & cruda & desaponificada \\
\hline Humedad & $9.60 \pm 0.02^{\mathrm{b}}$ & $13.59 \pm 0.01^{\mathrm{a}}$ \\
Grasa & $7.13 \pm 0.01^{\mathrm{a}}$ & $5.59 \pm 0.02^{\mathrm{b}}$ \\
Proteína & $15.60 \pm 0.01^{\mathrm{a}}$ & $15.53 \pm 0.01^{\mathrm{a}}$ \\
Ceniza & $3.20 \pm 0.01^{\mathrm{a}}$ & $2.52 \pm 0.01^{\mathrm{b}}$ \\
Fibra & $2.77 \pm 0.01^{\mathrm{a}}$ & $2.14 \pm 0.01^{\mathrm{a}}$ \\
Carbohidratos & $61.70 \pm 0.02^{\mathrm{a}}$ & $60.63 \pm 0.03^{\mathrm{b}}$ \\
\hline Letras distintas indican diferencia significativa entre las muestras
\end{tabular}

Tanto la quinua cruda, como la desaponificada cumplen con los requisitos bromatológicos de la legislación nacional ecuatoriana (Norma INEN 1673:2013). El proceso de desaponificación (lavado) produjo un incremento significativo en la humedad de los granos (aproximadamente un 30\%). Por el contrario, se observó una disminución del 20\% de grasas, cenizas y fibra y del $2 \%$ de carbohidratos. En cuanto al contenido proteico no se encontró diferencia significativa entre las muestras. Cervilla et al. (2014), indican que la inmersión de los granos de quinua en agua genera pérdida de nutrientes. 
Los beneficios de la quinua están relacionados con su alto valor nutricional debido a su contenido en proteínas y calidad de la misma. El contenido de proteína de la quinua oscila entre 13.81 y $21.9 \%$ según la variedad. Entre 16 y $20 \%$ del peso de la semilla de quinua corresponde a proteínas de alto valor biológico, que contienen todos los aminoácidos, incluidos los esenciales. La quinua se considera el único alimento vegetal que proporciona todos los aminoácidos esenciales, que están muy cerca de los estándares de nutrición humana establecidos por la FAO (2011). El balance de aminoácidos esenciales en la proteína de quinua es superior al trigo, cebada y soja, comparado favorablemente con la proteína de la leche. La quinua es rica en lisina, mientras que las proteínas de cereales son deficientes en este aminoácido (FAO, 2011).

Caracterización fisicoquímica de las cervezas. - La Tabla 4 muestra los resultados obtenidos para la densidad inicial y final (densidad medida antes y después de la fermentación), contenido alcohólico, pH del mosto y de la cerveza, acidez total y turbidez para cada formulación.

Tabla 4. Resultados obtenidos en la caracterización de los productos

\begin{tabular}{|c|c|c|c|c|}
\hline Formulación & QSM50 & QSM35 & QM50 & QM35 \\
\hline Densidad Inicial (g/cm3) & $1.051 \pm 0.002^{\mathrm{b}}$ & $1.053 \pm 0.003^{b}$ & $1.056 \pm 0.002^{\mathrm{a}}$ & $1.058 \pm 0.002^{\mathrm{a}}$ \\
\hline Densidad Final (g/cm3) & $1.011 \pm 0.002^{\mathrm{b}}$ & $1.013 \pm 0.002^{\mathrm{ab}}$ & $1.015 \pm 0.000^{\mathrm{ab}}$ & $1.016 \pm 0.002^{\mathrm{a}}$ \\
\hline Contenido alcohólico ('L) & $5.160 \pm 0.280^{\mathrm{b}}$ & $5.33 \pm 0.28^{b}$ & $5.50 \pm 0.00^{\mathrm{a}}$ & $5.66 \pm 0.28^{a}$ \\
\hline Turbic & $73.00 \pm 0.76^{b}$ & $58.00 \pm 1.00^{c}$ & $135.00 \pm 1.00^{\mathrm{a}}$ & $82.00 \pm 0.00^{b}$ \\
\hline $\begin{array}{l}\text { Cantidad }(\mathrm{ml}) \text { de } \mathrm{NaOH} 0.1 \mathrm{~N} \\
\text { utilizado para neutralizar }\end{array}$ & $9.20 \pm 0.14 a$ & $9.00 \pm 0.07 a$ & $8.50 \pm 0.28 b$ & $8.60 \pm 0.14 b$ \\
\hline Acidez Total (\% ácido láctico) & $0.160 \pm 0.140^{a}$ & $0.16 \pm 0.07^{a}$ & $0.15 \pm 0.28^{a}$ & $0.15 \pm 0.14^{a}$ \\
\hline pH mosto & $5.28 \pm 0.02^{a}$ & $5.25 \pm 0.35^{a}$ & $5.53 \pm 0.00^{\mathrm{a}}$ & $5.64 \pm 0.02^{\mathrm{a}}$ \\
\hline pH cerveza & $4.22 \pm 0.08^{\mathrm{a}}$ & 4.39. $\pm 0.04^{a}$ & $4.49 \pm 0.04^{\mathrm{a}}$ & $4.60 \pm 0.04^{a}$ \\
\hline
\end{tabular}

Letras diferentes indican diferencia significativa entre las muestras QSM: quinua sin maltear

QM: quinua malteada

La concentración y el malteado de la quinua influyeron sobre la densidad del mosto y de la cerveza. Las cervezas a base de quinua sin maltear empezaron la fermentación con menor densidad inicial. El proceso de malteado es la germinación controlada de los granos, seguida de la terminación de este proceso natural mediante la aplicación de calor (secado) para producir el sabor y el color requeridos. El proceso de secado produce compuestos esenciales de sabor y color, que son importantes para la calidad sensorial de la cerveza (Goode, Wijngaard y Arendt, 2005). El proceso de malteado solubiliza algunos componentes, como por ejemplo azúcares y proteínas, aumentando de esta forma la densidad inicial. El malteado tiene como objetivo generar en los granos las enzimas hidrolíticas endógenas las cuales descomponen los principales constituyentes químicos para proporcionar azúcares fermentables, nitrógeno libre, aminoácidos libres y otros. Por esta razón, la densidad inicial de los mostos elaborados es mayor cuando se utiliza quinua malteada. De la misma manera, si el mosto contiene mayor cantidad de materia fermentable, tendrá mayor contenido alcohólico al final de la etapa de fermentación (Tabla 4). El rango de densidades finales en cervecería oscila entre $0.997-1.040 \mathrm{~g} / \mathrm{mL}$ según el tipo de material amiláceo utilizado (Rodríguez, 2003).

Según García et al. (2004), la relación de conversión de carbohidratos a alcohol se maneja en cervecería como atenuación con valores típicos de $70-80 \%$, actualmente se estudia el uso de cepas capaces de producir glucoamilasa o pululanasa para transformar las dextrinas en azúcares fermentables. La diferencia de contenido alcohólico fue causada principalmente por el aporte de azúcares de la quinua malteada. En las formulaciones realizadas con quinua sin maltear con densidades iniciales y finales inferiores, se evidenció un contenido alcohólico menor que las realizadas con quinua malteada. La densidad está 
estrictamente relacionada con la cantidad de alcohol producida en la cerveza, mientras se van transformando los azúcares en alcohol, la bebida se hace más ligera. La densidad inicial y final se puede utilizar también para relacionar el consumo de azúcares realizado por las levaduras y su posterior conversión en alcohol y dióxido de carbono (Rodríguez, 2003). Además, la diferencia puede ser debido a la limitada actividad enzimática del mosto o la presencia de inhibidores enzimáticos en la quinua sin maltear (Elizalde, Portilla, y Chaparro, 2009).

González y Muñoz (2000) señalan que una cerveza tipo Ale de calidad debe presentar un valor de densidad entre $0.998-1.018 \mathrm{~g} / \mathrm{ml}$ y un contenido de alcohol entre 2.5 a $9 \%$. Asimismo, la norma técnica ecuatoriana INEN 2262 indica que las cervezas deben contener máximo $10^{\circ} \mathrm{GL}$, validando los resultados obtenidos en este estudio.

Según los autores Goode, Wijngaard y Arendt (2005) y Lowe et al., (2004) el uso de cebada no malteada da como resultado bajos rendimientos de extracto, alta viscosidad del mosto, una disminución en la velocidad de pulverización y la formación de una neblina indeseable, así como impactos negativos sobre el sabor de la cerveza, en comparación con cerveza elaborada con $100 \%$ de cebada malteada.

En cuanto a la acidez, las 4 formulaciones presentan valores menores a $0.3 \%$ de ácido láctico, cumpliendo con el valor máximo permitido por la norma INEN 2262 (Tabla 4). Las cervezas elaboradas a base de quinua sin maltear obtuvieron una acidez ligeramente mayor que las elaboradas a base de quinua malteada, sin encontrarse diferencia significativa entre las muestras debido a que el proceso fermentativo fue controlado durante 7 días para todas las cervezas. La acidez resultante de los 4 productos se considera aceptable y no influyó en el sabor, en tanto que, si la fermentación habría sido extendida por más de 7 días, el proceso fermentativo habría generado mayor acidez y características de aroma y sabor indeseables por los consumidores (Kunze, 2006).

Un pH elevado es desfavorable en la sacarificación ya que influye en la actividad enzimática provocando menor cantidad de azúcares. Durante la maceración y filtración una disminución de la coagulación de las proteínas generan mayor turbidez y amargor por extracción de abundantes taninos (polifenoles) y riesgos microbiológicos adicionales (Rodríguez, 2003). Los valores de $\mathrm{pH}$ resultantes de los mostos y de las cervezas elaboradas a base de quinua malteada fueron ligeramente mayores que las elaboradas a base de quinua sin maltear, sin encontrarse diferencia estadística. Luego de la sacarificación el mosto registró un pH considerado óptimo en cervecería, entre 5.1 a 5.2 (Holle, 2003); y luego del proceso fermentativo el $\mathrm{pH}$ de todas las formulaciones se redujo por la acidez generada por este proceso. Los valores obtenidos cumplen según la norma técnica ecuatoriana INEN 2262.

Además, de entre todos los atributos de la cerveza, el color es uno de los más interesantes ya que es el principal atributo del aspecto de la misma. El color de la cerveza se debe principalmente a las maltas utilizadas. El color viene determinado por la temperatura de secado durante el malteado, de forma que temperaturas más altas producen maltas más oscuras (Rodríguez, 2003). El malteado de la quinua influyó directamente en los parámetros de color. Los valores de luminosidad, de las coordenadas $a^{*}$ y b*, del ángulo Hue y del croma de las cervezas se muestran en la Tabla 5. Las cervezas elaboradas con quinua sin maltear presentaron valores mayores de luminosidad con una coloración más clara que las cervezas con quinua malteada, las cuales por el contrario obtuvieron menor luminosidad. La quinua malteada le infirió un color ligeramente ámbar a las cervezas por el proceso de secado y posterior tostado en el malteado, mientras que las cervezas con quinua sin maltear presentaron una tonalidad más amarilla.

El color obtenido en las maltas está relacionado con la aplicación de calor y las reacciones que ocurren en esa etapa, como las reacciones de cambios de color no enzimáticas que comprometen a los carbohidratos y proteínas, como la reacción de Maillard y la caramelización (Steiner et al., 2012). De acuerdo con Briggs et al. (2004), el color de la cerveza en gran parte está relacionado con la melanoidina, compuestos de la caramelización y compuestos fénolicos presentes en la malta y puede verse afectado por 
el contenido de pigmentos naturales presentes en la quinua como son los compuestos fenólicos y betalaínas (Yao et al., 2015, Abderrahim et al., 2015).

Tabla 5. Análisis de color de las cuatro formulaciones de cerveza

\begin{tabular}{lccccc}
\hline Formulación & $\mathbf{L}^{*}$ & $\mathbf{a}^{*}$ & $\mathbf{b}^{*}$ & Hue & Croma \\
\hline QSM50 & $46.21 \pm 1.06^{\mathrm{a}}$ & $4.42 \pm 0.16^{\mathrm{b}}$ & $10.88 \pm 0.88^{\mathrm{ab}}$ & $57.48^{\mathrm{ab}}$ & $11.74^{\mathrm{ab}}$ \\
QSM35 & $46.88 \pm 0.11^{\mathrm{a}}$ & $4.42 \pm 0.02^{\mathrm{b}}$ & $12.64 \pm 0.10^{\mathrm{a}}$ & $59.40^{\mathrm{a}}$ & $13.39^{\mathrm{a}}$ \\
QM50 & $43.83 \pm 0.67^{\mathrm{b}}$ & $4.58 \pm 0.55^{\mathrm{a}}$ & $9.64 \pm 1.28^{\mathrm{b}}$ & $55.42^{\mathrm{b}}$ & $10.67^{\mathrm{b}}$ \\
QM35 & $41.12 \pm 0.16^{\mathrm{c}}$ & $4.64 \pm 0.05^{\mathrm{a}}$ & $7.53 \pm 0.87^{\mathrm{c}}$ & $51.86^{\mathrm{c}}$ & $8.84^{\mathrm{c}}$ \\
\hline
\end{tabular}

Letras diferentes indican diferencia significativa entre las muestras

QSM: quinua sin maltear

QM: quinua malteada

La claridad de la cerveza también está considerada como un parámetro de mucha importancia en la degustación de la misma. La medición de turbidez de las 4 formulaciones de cerveza demostró que las formulaciones obtenidas con quinua malteada generaron un líquido con mayor cantidad de partículas en suspensión que las cervezas elaboradas con quinua sin maltear (Tabla 6). La menor turbidez de todas las cervezas se obtuvo con el menor porcentaje de quinua sin maltear (35\%). Por el contrario, la mayor turbidez de todas se produjo en la cerveza elaborada con el mayor porcentaje $(50 \%)$ de quinua malteada. Se puede decir entonces que tanto el malteado y así como la cantidad de quinua y cebada utilizada en cada formulación tienen una influencia directa en la turbidez de la cerveza. En la etapa de malteado, el proceso de germinación causa ciertos cambios en los constituyentes químicos de los granos generando partículas de los componentes de menor tamaño (Goode, Wijngaard y Arendt, 2005).

La turbidez es un problema importante en la producción de cerveza, ya que afecta la calidad del producto final. La cerveza consiste en varios ingredientes tales como proteínas, carbohidratos, polifenoles, ácidos grasos, ácidos nucleicos, aminoácidos, cristales de oxalatos, polifenoles, almidones, fibras como $\beta$-glucano, pentosanos, entre otros (Steiner, 2010), que pueden precipitar y producir turbidez. Brunelli, Mansano y Venturini (2014) reportaron que, con la adición de miel a la formulación de cerveza, estas presentan menor grado de turbidez, indicando que la cebada malteada es la principal fuente de sustancias coloidales como proteínas, polisacáridos y polifenoles. Además, la quinua en su composición presenta también un alto contenido de sustancias coloidales como almidón, fibras y proteínas (Guantian y Fan, 2017), lo cual puede provocar el aumento de la turbidez en la cerveza.

Análisis Sensorial. - En la Tabla 6 se presentan los resultados obtenidos del análisis sensorial realizado a las 4 formulaciones diferentes de cerveza. La cerveza con $50 \%$ de quinua malteada (QM50) presentó los valores más altos en los atributos de transparencia, color, cuerpo, sabor y aceptación global. En relación con los análisis físico-químicos puede decirse que el proceso de malteado proporciona precursores del sabor y ayuda en la obtención de una cerveza con mejores características sensoriales (mejor sabor, cuerpo, color y aceptación global). Resultados similares han sido publicados en cuanto a una buena aceptación de cervezas elaboradas con materias primas alternativas como camote morado (Panda et al., 2015) y arroz (Mayer et al., 2016), no obstante, son necesarios más estudios sobre el desarrollo de perfiles sensoriales que relacionen con el sabor y color adecuados de las cervezas (Goode, Wijngaard \& Arendt, 2005).

\section{Conclusiones}

Los procesos de desaponificación y malteado de la quinua influyeron directamente en el valor nutricional, densidad, contenido alcohólico, color y turbidez de las 4 formulaciones de cerveza. Debido a la desaponificación de la quinua el porcentaje de 
humedad aumentó y por el contrario se produjo una disminución en el contenido de carbohidratos, grasa, cenizas y fibra. La densidad fue más elevada en las formulaciones a base de quinua malteada respecto a las cervezas elaboradas con quinua sin maltear. La quinua malteada generó un color más intenso, menor luminosidad y mayor turbidez, además de contenidos alcohólicos más elevados. El tratamiento con mayor aceptación global fue elaborado a partir de un $50 \%$ de quinua malteada, el cual superó a las demás formulaciones no solo en aceptación global, sino también en transparencia, color, cuerpo y sabor. El uso de quinua malteada para la elaboración de cerveza constituye una alternativa para el procesamiento de este cereal y un producto novedoso para el consumidor.

Tabla 6. Análisis sensorial de 4 formulaciones de cerveza

\begin{tabular}{lcccc}
\hline \multicolumn{1}{c}{ Atributo } & QSM50 & QSM35 & QM50 & QM35 \\
\hline Transparencia & $6.85 \pm 1.90^{\mathrm{b}}$ & $6.95 \pm 1.79^{\mathrm{b}}$ & $7.28 \pm 1.65^{\mathrm{a}}$ & $6.82 \pm 1.98^{\mathrm{b}}$ \\
Color & $7.68 \pm 1.52^{\mathrm{a}}$ & $7.57 \pm 1.62 \mathrm{a}^{\mathrm{b}}$ & $7.73 \pm 1.54^{\mathrm{a}}$ & $7.48 \pm 1.63^{\mathrm{b}}$ \\
Aroma & $7.27 \pm 1.74^{\mathrm{a}}$ & $6.97 \pm 1.87^{\mathrm{b}}$ & $6.99 \pm 1.81^{\mathrm{b}}$ & $6.93 \pm 1.91^{\mathrm{b}}$ \\
Cuerpo & $7.16 \pm 1.56^{\mathrm{b}}$ & $7.02 \pm 1.65^{\mathrm{b}}$ & $7.36 \pm 1.42^{\mathrm{a}}$ & $7.14 \pm 1.79^{\mathrm{b}}$ \\
Sabor & $6.95 \pm 1.83^{\mathrm{b}}$ & $6.56 \pm 1.79^{\mathrm{c}}$ & $7.03 \pm 1.86^{\mathrm{a}}$ & $6.94 \pm 1.95^{\mathrm{b}}$ \\
Sabor residual & $7.14 \pm 1.82^{\mathrm{a}}$ & $6.45 \pm 1.93^{\mathrm{c}}$ & $6.94 \pm 1.81^{\mathrm{b}}$ & $7.01 \pm 1.96^{\mathrm{b}}$ \\
Aceptación global & $7.24 \pm 1.47^{\mathrm{b}}$ & $6.85 \pm 1.57^{\mathrm{c}}$ & $7.51 \pm 1.45^{\mathrm{a}}$ & $7.25 \pm 1.50^{\mathrm{b}}$ \\
\hline
\end{tabular}

Letras minúsculas en la misma fila indican diferencia significativa QSM: quinua sin maltear QM: quinua malteada

\section{Bibliografía}

Abderrahim, F., Huanatico, E., Segura, R., Arribas,S., González, M. C., y Condezo-Hoyos, L. (2015). "Physical features, phenolic compounds, betalains and total antioxidant capacity of coloured quinoa seeds (Chenopodium quinoa Willd.) from Peruvian Altiplano". Food Chemistry, 183(15), p. 83-90. https://doi.org/10.1016/j.foodchem .2015.03.029

Agu, R. C., Palmer, G. H. (1996). "Enzymatic breakdown of endosperm proteins of sorghum at different malting temperatures". Journal of the Institute of Brewing, 102(6), p. 415418. Doi:10.1002/j.2050-0416.1996.tb00924.x.

AOAC. (2000). Official methods of analysis of AOAC.

Bamforth, C. (2003). Beer -Tap Into the Art and Science of Brewing Second edition. New york: Oxford.

Boffill-Rodríguez, Y., y Gallardo-Aguilar, I. (2014). "Ventajas de la producción de cerveza a partir de malta de sorgo: Revisión bibliográfica". Tecnología Química, 34(3), 324-334.

Bravo, M., Reyna, J., Gómez, I., y Huapaya, M. (2013). "Estudio químico y nutricional de granos andinos germinados de Quinua" (Chenopodiun quinoa) y Quiwicha (Amarantus Cudatus). Revista Peruana de Química e Ingeniería química, 16(1), 5460.

Brunelli, L. T., Mansano, A. R., y Venturini Filho, W. G. (2014). "Caracterização físicoquímica de cervejas elaboradas com mel". Brazilian Journal of Food Technology, 17(1), 19-27. http://dx.doi.org/10.1590/bjft.2014.004.

Cervilla, N., Mufari, J., Calandri, E., y Guzmán, C. (2014). "Pérdidas Nutricionales durante la cocción de semilla de chenopodium quinoa wild bajo presión de vapor". Cordova, Argentina: Instituto de Ciencias y Tecnología de los Alimentos.

Daniels, R. (2000). Designing Great Beers. Brewers Publications. Boulder, CO.

De Bruin, A. (2006). "Investigation of the food value of quinua and canihua seed". Journal of Food Science, 29(6), p. 872-876. Doi:10.1111/j.1365-2621.1964.tb00464.x

Elizalde, A. D., Portilla, Y. P., y Chaparro, D. C. C. (2009). "Factores antinutricionales en 
semillas". Biotecnología en el Sector Agropecuario y Agroindustrial, 7(1), 45-54.

FAO. (2011). Quinoa: an Ancient Crop to Contribute to World Food Security. Recuperado de http://www.fao.org/quinoa-2013/publications/detail/en/item/202738/icode/ (accedido el 12/10/2017).

FAO. (2013). Organización Para las Naciones Unidas Para la Agricultura y la Alimentación: Año Internacional de La Quinua. Recuperado de http://www.fao.org/quinoa-2013/es/ (accedido el 12/10/2017).

FAO. (2014). Tendencias y perspectivas del comercio internacional de la quinua. Santiago, Chile. Recuperado de http://www.fao.org/3/a-i3583s.pdf (accedido el 12/10/2017).

García Garibay, M., Quintero Ramírez, R., y López Mungía, A. (2004). "Biotecnología Alimentaria". Limusa, S.A. México.

García, M., Quinteros, R., y López, A. (1993). "Biotecnología Alimentaria". Limusa. S.A. México.

González, L. y Muñiz, P. (2000). "Actividad antioxidante de la cerveza: estudios in vitro". Universidad de Burgos. Burgos, España.

Guantian, L. y Fan, Z. (2016). "Physicochemical properties of quinoa flour as affected by starch interactions". Food Chemistry, 221(15), 2017, p.1560-1568. https://doi.org/10.1016/j.foodchem.2016.10.137

INEC. (2011). Instituto Ecuatoriano de Estadística y Censos. Bebidas alcohólicas. Ecuador en cifras. 2011.

Koziol, M. J. (1992). "Chemical composition and nutritional evaluation of quinoa (Chenopodium quinoa Willd.)". Journal of Food Composition and Analysis, 5(1), 3568. https://doi.org/10.1016/0889-1575(92)90006-6

Kunze, W. (2006). "Tecnología para cerveceros y malteros". Berlín, Alemania: WestkreuzDruckerei Ahrens KG.

Mattos, R. (2004). "Tecnología cervejeira". Campinas - SP: Instituto de Tecnología de Alimentos. Brasil: ITAL.

Mayer, H., Ceccaroni, D., Marconi, O., Sileoni, V., Perretti, G., Fantozzi, P. (2015). "Development of an all rice malt beer: a gluten free alternative", LWT - Food Science and Technology. 67, p. 67-73. doi: 10.1016/j.Iwt.2015.11.037.

Monteros Guerrero, A. (2016). "Rendimientos De Quinua En El Ecuador 2016". Dirección de Análisis y Procesamiento de la Información Coordinación General del Sistema de Información Nacional Ministerio de Agricultura, Ganadería, Acuacultura y Pesca Quito, Ecuador.

Nieto, C., y Vimos, C. (1998). "La Quinua, cosecha y postcosecha. Algunas experiencias en Ecuador". Quito: INIAP.

NTE INEN 1672:2013. Instituto Ecuatoriano de Normalización. Quinua. Determinación de contenido de saponinas por medio del método espumoso. Quito, Ecuador.

NTE INEN 1673:2013. Instituto Ecuatoriano de Normalización. Quinua. Requisitos. Quito, Ecuador.

NTE INEN 2262. 2013. Instituto Ecuatoriano de Normalización. Bebidas alcohólicas. Cerveza. Requisitos. Quito, Ecuador.

Olsen, E. (2003). "Métodos Ópticos de Análisis". Editorial Reverte.

Panda, S.K.; Panda, S.H.; Swain, M.R., Ray, R.C. y Kayitesi, E. (2015). "Anthocyanin-Rich Sweet Potato (Ipomoea batatas L.) Beer: Technology, Biochemical and Sensory Evaluation". Journal of food processing and preservation, 39(6), p. 2040-3049. Doi:10.1111/jfpp.12569.

Przybylski, R., Chauhan G., y Eskin N. (1994). Characterization of quinoa (Chenopodium quinoa) lipids. Food Chemistry, 51(2), p. 187-192. https://doi.org/10.1016/03088146(94)90255-0.

Repo-Carrasco, R., Espinoza, C., y Jacobsen, S. E. (2003). "Nutritional value and use of the andean crops quinoa (Chenopodium quinoa) and kaniwa (Chenopodium pallidicaule)". Food Reviews International, 19(1), p. 179 y 189. http://dx.doi.org/10.1081/FRI-120018884. 
Rodríguez, A. (2016). El ecuatoriano consume apenas media libra de quinua al año. Diario El Telégrafo. Recuperado de https://www.eltelegrafo.com.ec/noticias/septimo/1/elecuatoriano-consume-apenas-media-libra-de-quinua-al-ano (accedido 15/10/2017).

Rodríguez, H. (2003). "Determinación de parámetros fisicoquímicos para la caracterización de cerveza tipo Lager elaborada por compañía cervecera Kunstmann S.A." Tesis para optar el grado académico de Licenciado en Ingeniería en Alimentos. Universidad Austral de Chile. Valdivia, Chile.

Steiner, E., Auer, A., Becker, T., y Gastl, M. (2012). "Comparison of beer quality attributes between beers brewed with $100 \%$ barley malt and $100 \%$ barley raw material". Journal of the Science of Food and Agriculture, 92(4), p. 803-813. Doi:10.1002/jsfa.4651.

Steiner, E., Becker, T., y Gastl, M. (2010). "Turbidity and haze formation in beer- Insights and overview". Journal of the Institute of Brewing, 116, 360-368.

Tang, Y., Li, X., Zhang, B., Chen, P., Liu, R., y Tsao, R. (2015). "Characterisation of phenolics, betanins and antioxidant activities in seeds of three Chenopodium quinoa Willd. Genotypes". Food Chemistry, 166(1), 380-388. https://doi.org/10.1016 /j.foodchem.2014.06.018.

Zainashef, J., y Palmer, J. (2007). "Brewing Classic Styles". Boulder, Colorado: Brewers Publications. 